\title{
Terahertz Rotational Spectroscopy
}

\author{
T. F. Giesen ${ }^{1}$, S. Brünken ${ }^{2}$, M. Caris ${ }^{1}$, P. Neubauer-Guenther ${ }^{1}$, \\ U. Fuchs ${ }^{1}$, G. W. Fuchs ${ }^{3}$ and F. Lewen ${ }^{1}$ \\ ${ }^{1}$ I. Physikalisches Institut, Universität zu Köln, Zülpicher Str. 77, 50937 Cologne, Germany \\ ${ }^{2}$ Harvard-Smithsonian Center for Astrophysics, \\ 60 Garden Street, Cambridge, MA 02138, USA \\ ${ }^{3}$ Raymond and Beverly Sackler Laboratory for Astrophysics, Leiden Observatory, \\ Leiden University, Postbus 9513, 2300 RA, Leiden, The Netherlands
}

\begin{abstract}
The detection of interstellar molecules relies on the precise knowledge of spectral line positions from laboratory measurements. Technical developments of recent years have led to an extension of the accessible spectral range towards shorter wavelengths. New telescopes like SOFIA, the HIFI instrument aboard the Herschel satellite, and ALMA will be used for astrophysical observations in the terahertz region. The Cologne group has developed precise spectrometers to study molecules of astrophysical importance under laboratory conditions and to obtain characteristic spectra for their possible detection in space. We present recent results on light hydrides, carbon-chain molecules and more complex species.
\end{abstract}

Keywords. ISM: molecules — methods: laboratory

\section{Introduction}

Radioastronomy has been quite successful over the past decades in detecting new molecules in space. So far, more than 130 species have been clearly identified by means of characteristic spectral lines, and the number of new detections grows on average by four per year. While most of the detections were made at centimeter- and millimeterwavelengths, new receiver technologies are opening up the spectral range towards shorter wavelengths and leading into the sub-millimeter wavelength region, also known as the terahertz domain. A number of new telescopes, such as the Atacama Pathfinder Experiment, APEX, the Stratospheric Observatory for Infrared Astronomy, SOFIA, and the HIFI instrument aboard the Herschel satellite will explore the terahertz domain and will certainly lead to the detection of new interstellar molecular species. This identification of new molecules relies on precise laboratory spectra. It is, thus, a challenging task for laboratory groups world wide to provide precise spectral data of molecules which might be of astrophysical importance.

Over the last two decades, the Cologne group has developed precise spectrometers to cover the spectral range from $50 \mathrm{GHz}$ to $2.7 \mathrm{THz}$. In this paper we emphasize particular investigations of molecules that have characteristic spectra in the terahertz region, and which can be assigned to three important classes:

(1) pure rotational spectra of light hydrides,

(2) low ro-vibrational bending modes of carbon-chain molecules,

(3) spectra of large molecules with internal motions.

We will discuss these three aspects of terahertz spectroscopy by giving examples of molecules, which have recently been studied at the Cologne laboratories, and which are of particular interest for astronomy. In $\S 2$ we present our recent results on the methylene radical, $\mathrm{CH}_{2}$, a light hydride having pure rotational transitions from the lowest energy 


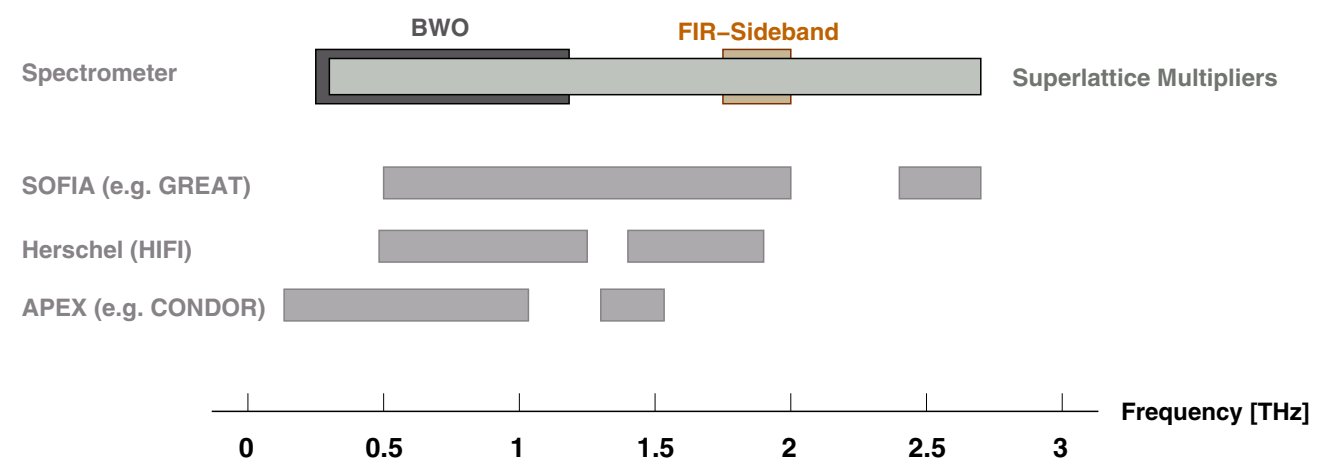

Figure 1. Frequency coverage of the Cologne spectrometers and the observatories SOFIA, Herschel and APEX.

levels at 2-3 THz. Ro-vibrational bending mode transitions of many molecules occur predominantly in the mid-infrared region, whereas carbon chains possess unusually low frequency bending vibrations. For example, the $\nu_{2}$ mode of $\mathrm{C}_{3}$ has been found at $2 \mathrm{THz}$ and high-level $a b$ initio calculations predict the lowest vibrational modes of longer pure carbon chains, such as $\mathrm{C}_{4}, \mathrm{C}_{5}, \ldots, \mathrm{C}_{10}$ to lie in the range of $1-5 \mathrm{THz}$. In $\S 3$ we discuss the spectroscopy of carbon-chain molecules and present recent results on $\mathrm{C}_{3} \mathrm{H}$. $\S 4$ deals with the spectral complexity of larger molecules and recent results on ethyl methyl ether, $\mathrm{C}_{2} \mathrm{H}_{5} \mathrm{OCH}_{3}$. $\S 5$ contains some general remarks on future spectroscopic investigations in the $\mathrm{THz}$ region and on the necessity of a data base for molecular spectroscopy.

The Cologne THz-spectrometers use backward wave oscillators (BWOs) as radiation and fundamental frequency sources to cover the frequency range from $50 \mathrm{GHz}$ to $1.1 \mathrm{THz}$. Two different approaches are followed to extend the accessible frequency range beyond this point. First, the BWO output radiation is mixed with the radiation of a fixed frequency far infrared laser to generate sideband radiation between $1.75-2.01 \mathrm{THz}$. Secondly, to cover the gap between $1-2 \mathrm{THz}$ and, furthermore, to access frequencies up to 2.7 THz, efficient superlattice multiplier devices (Scheuerer et al. 2003), pumped by the radiation of BWOs at $100-300 \mathrm{GHz}$ are used. Figure 1 shows the spectral coverage of our spectrometers and of some present and future telescope receivers.

High spectral accuracy, $\Delta f / f=10^{-11}$, is obtained by phase-locking the BWO to a Rubidium reference, and spectra can be recorded with Doppler- and partially sub-Doppler limited resolution. Detailed descriptions of the spectrometers can be found elsewhere (Winnewisser et al. 1994). The spectrometers have been combined with several devices for the production of unstable and highly-reactive molecules. These molecular sources include discharge cells, radio plasma techniques, and supersonic jets.

\section{Light Hydrides - The Methylene Radical, $\mathrm{CH}_{2}$}

Large rotational constants are characteristic for light hydrides, defined here as molecules of the form $\mathrm{XH}_{n}(\mathrm{n}=1,2)$. Consequently, even their ground state rotational transitions appear at comparatively high frequencies. At Cologne, the rotational spectra of a multitude of light hydride species have been measured during the last years by employing the Cologne terahertz spectrometers. Among these are the water molecule, $\mathrm{H}_{2} \mathrm{O}$, (Brünken et al. 2005), the $\mathrm{NH}$ radical (Lewen et al. 2004), the amidogen radical $\mathrm{NH}_{2}$ (Gendriesch et al. 2001), and the phosphidogen radical $\mathrm{PH}_{2}$ (Margulès et al. 2002). 
The methylene radical, $\mathrm{CH}_{2}$, is an important molecule for both spectroscopists and astrochemists. It is expected to be abundant in both dense and diffuse interstellar clouds and to be presumably produced quite early in the sequence of ion-molecule reactions, which makes it a test molecule for chemical models (Lee et al. 1996). Due to its lightness, the lowest levels are already high in energy and are widely spaced in energy. Thus transitions which probe the lowest rotational states occur in the terahertz spectral region. In its electronic ${ }^{3} B_{1}$ ground state it has a bent, symmetric structure with an $\mathrm{H}-\mathrm{C}-\mathrm{H}$ equilibrium bond angle of 133.9308(21) degrees (Jensen \& Bunker 1988) and a low barrier to linearity of less than $2000 \mathrm{~cm}^{-1}$. A very shallow potential energy curve causes its floppiness, which in turn leads to strong centrifugal distortion effects. The approach of a standard Watson-type $A$-reduced Hamiltonian fails in the case of light and floppy molecules and leads to large uncertainties for the prediction of transition frequencies, especially at higher rotational quantum numbers.

$\mathrm{CH}_{2}$ is an asymmetric top molecule with a permanent dipole moment of 0.57 Debye (Bunker \& Langhoff 1983) pointing along the $b$ molecular axis, which is the bisecting line of the $\mathrm{H}-\mathrm{C}-\mathrm{H}$ angle. Although $\mathrm{CH}_{2}$ is a simple triatomic molecule, its spectrum is fairly complicated due to fine and hyperfine interactions. The electron spin in a ${ }^{3} B_{1}$ ground state $(\mathrm{S}=1)$ causes a splitting of each rotational energy level into three fine structure components. Due to the presence of two protons, $\mathrm{CH}_{2}$ has both an ortho (total nuclear spin, $\mathrm{I}=1)$ and a para $(\mathrm{I}=0)$ form. In the case of ortho- $\mathrm{CH}_{2}$, the nuclear spin causes a further splitting of each fine structure rotational level into three hyperfine levels.

The first laboratory measurements on $\mathrm{CH}_{2}$ were performed by Herzberg \& Shoosmith (1959), which led to a clear identification of the ${ }^{3} B_{1}$ ground state and later to the determination of its structure. In the beginning of the 1980s, Sears et al. used laser magnetic resonance spectroscopy to record the first ro-vibrational spectra in the midinfrared (Sears et al. 1981, 1982) and pure rotational spectra of the vibrational ground state in the far infrared region (Sears et al. 1982a). These data were used by Lovas et al. (1983) to perform the first measurements on the $4_{04}-3_{13}$ pure rotational transition at $\sim 70 \mathrm{GHz}$ with microwave accuracy. Hollis et al. (1989) used the $70 \mathrm{GHz}$ transition to start several attempts for an interstellar detection of $\mathrm{CH}_{2}$ towards the Orion nebula. The tentative detection was later confirmed by the same authors using a receiver of improved sensitivity (Hollis et al. 1995). Ozeki \& Saito (1995) measured two rotational transitions of para- $\mathrm{CH}_{2}$, which correspond to excitation temperatures as high as $135 \mathrm{~K}$ and $310 \mathrm{~K}$ respectively. A search for $\mathrm{CH}_{2}$ in the cold interstellar medium requires precise data of transitions, where the lowest energy levels are involved. Because of its floppiness these data can not be obtained to high accuracy from predictions based on a standard Watson Hamilton approach. To overcome these constraints, we started extensive laboratory studies on $\mathrm{CH}_{2}$ in 2003 with the clear intention of extending the sparse data set towards terahertz frequencies and supporting a decisive astrophysical search for $\mathrm{CH}_{2}$ in cold interstellar clouds.

We produced the methylene radical from ketene, $\mathrm{CH}_{2} \mathrm{CO}$, which was disintegrated in a DC-discharge cell cooled to a temperature of roughly $170 \mathrm{~K}$ to populate the lower-energy levels of $\mathrm{CH}_{2}$. A $4 \mathrm{kHz}$ Zeeman modulation was employed for phase sensitive signal detection, making use of the permanent magnetic moment of methylene. We used the Cologne $\mathrm{THz}$ spectrometer for measuring the hyperfine components of the $N_{K a, K c}=1_{1,1}-2_{0,2}$ transition of ortho- $\mathrm{CH}_{2}$ centered at $943 \mathrm{GHz}$ (Michael et al. 2003) and recorded two energetically low-lying transitions near $2 \mathrm{THz}$ (Brünken et al. 2004) by operating the Cologne Sideband Spectrometer for Terahertz Applications (COSSTA; Lewen et al. 1997). One of those transitions, the $N_{K a K c}=1_{1,0}-1_{0,1}$ at $1.915 \mathrm{THz}$ stems from the lowest para energy level and is best suited for a detection of $\mathrm{para}-\mathrm{CH}_{2}$ in a cold interstellar 
environment. To fit the data to an effective Hamiltonian, we used a new approach introduced by Pickett et al. (2005), which is based on an expansion of the angular momentum operators in terms of an Euler series. All our data and all available precise data from the literature were included in the fit to give the most reliable set of molecular constants available (Brünken et al. 2005a). Based on this analysis, transition frequencies from the lowest energy levels of $\mathrm{CH}_{2}$ were calculated and used by Polehampton et al. (2005) to search for $\mathrm{CH}_{2}$ in the cold interstellar medium. For this purpose, the authors analyzed data obtained with the Long Wavelength Spectrometer (LWS; Clegg et al. 1996) aboard the Infrared Space Observatory (ISO). Several fine structure components of three rotational transitions of $\mathrm{CH}_{2}$ were detected in cold molecular clouds in the line of sight towards the galactic center source Sagittarius B2 (Sgr B2). These transitions involve the three lowest rotational energy levels of methylene. The detections allowed the determination of the $\mathrm{CH}_{2}$ column density and the estimation of the ortho/para ratio in the observed regions. Furthermore, the simultaneous observation of $\mathrm{CH}$ and $\mathrm{CH}_{2}$ absorption lines gave insight into the underlying chemistry by comparison of the measured abundance ratio to that derived from different theoretical models. These astrophysical investigations were triggered by and based upon state-of-the-art laboratory measurements and their subsequent analysis.

\section{Low Bending Modes of Carbon-Chain Molecules}

Carbon is the fourth most abundant element in space. A large fraction of the more than 130 different interstellar molecules contain at least one carbon atom. Due to its unique ability to form linear chains, rings and branched, three dimensional structures, carbon is the most important element of a complex interstellar chemistry. It is conspicuous that many interstellar detected molecules are highly unsaturated linear carbon chains. Chemical models also predict pure carbon chains to be quite abundant in interstellar clouds and in the environment of late type carbon stars; nevertheless, only $\mathrm{C}_{3}$ and $\mathrm{C}_{5}$ have been undoubtedly detected so far. In the 19th century, Huggins (1881) discovered a spectral feature in the optical spectrum of comet b 1881 which was later assigned to $\mathrm{C}_{3}$ by Douglas (1951). Based on ro-vibrational transitions in the infrared Hinkle et al. (1988) detected $\mathrm{C}_{3}$ in the shell of the carbon star IRC +10216 . Recent detections by rotationally resolved spectra in the visible range were published by Maier et al. (2001), who found $\mathrm{C}_{3}$ in three diffuse interstellar clouds towards reddened stars and by Adamkovics et al. (2003) in translucent sight lines. The only secure detection of $\mathrm{C}_{5}$ was reported by Bernath et al. (1989) towards IRC+10216 based on infrared spectra.

The main reason for the almost complete absence of pure carbon chains from the list of interstellar molecules is the missing of a permanent dipole moment, which would allow their detection by pure rotational transitions in the millimeter-wave region. However, pure carbon chains are predicted to have low-lying bending vibrations in the terahertz region. Precise knowledge of these transition frequencies would enable astronomers to search for longer linear carbon chains in cold interstellar clouds. Ab initio calculations on the structure and the vibrational frequencies were performed by e.g. Martin \& Taylor (1996) and Botschwina (2005) by means of coupled cluster calculations. The bending mode vibrational dipole moment is on the order of $0.2-0.3$ Debye for most of the $\mathrm{C}_{n}$ chains with $n=3-14$.

$\mathrm{C}_{3}$ is the only pure carbon-chain molecule for which measurements of the lowest bending mode transitions have been performed, using tunable far-infrared laser sideband spectroscopy at $2 \mathrm{THz}$ (Schmuttenmaer et al. 1990; Gendriesch et al. 2003). The measurements by Schmuttenmaer et al. led to the detection of $\mathrm{C}_{3}$ in the source Sgr B2, 


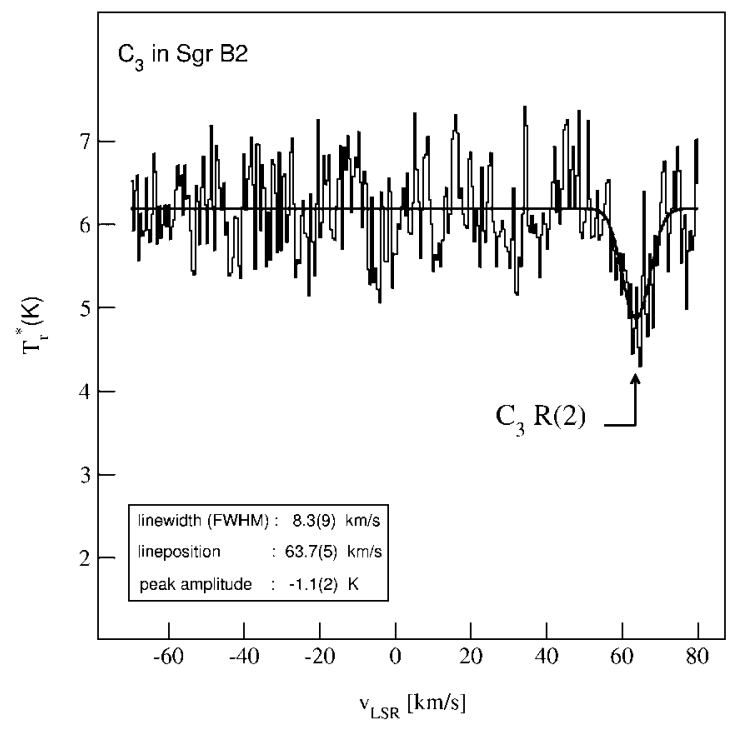

Figure 2. $\mathrm{C}_{3}$ absorption line toward Sgr B2 using the KAO. The peak was assigned to the $\mathrm{R}(2)$ transition of the $\nu_{2}$ bending mode.

which was the first detection of a pure carbon molecule in an interstellar cloud at all (Van Orden et al. 1994 and Giesen et al. 2001; see Fig. 2). This one-line detection was later confirmed by Cernicharo et al. (2000), using the long wavelength Fabry-Perot spectrometer (LWS) aboard the ISO satellite to detect $\mathrm{C}_{3}$ in Sgr B2 and IRC+10216. The terahertz region will be discovered by a new generation of telescopes, such as SOFIA and the HIFI instrument aboard the Herschel satellite. Due to the lack of accurate laboratory data, it is inevitable to perform high-resolution measurements on the bending modes in this spectral region. For this purpose we have combined our supersonic jet molecular sources with a new Terahertz-Spectrometer. Figure 3 shows the experimental setup. The Supersonic Jet Spectrometer for Terahertz Applications (SuJeSTA) is an instrument that allows high-resolution mm- and submm-wave spectroscopy of unstable, highly-reactive molecules, such as radicals and ions at low rotational temperatures of a few Kelvin. Highly-reactive molecules are produced in either an electrical discharge, placed in the throat of the source, or by laser ablation of a graphite target. Both techniques are well established and have been described in various papers (see e.g. Linnartz et al. 2000; Giesen et al. 1994).

A variety of molecules can be produced by an appropriate mixture of gases entering the discharge source. The jet is probed by the radiation of tunable BWOs operating between 300 and $1200 \mathrm{GHz}$ and very recently in combination with superlattice devices up to $2.7 \mathrm{THz}$. The spectrometer can be operated in two modes, a frequencystabilized mode for highly-precise measurements over a small range of up to $500 \mathrm{MHz}$ (typically 10-20 MHz), or in a free-running fast-scanning mode, which covers a large frequency range of several $\mathrm{GHz}$ but with less accuracy. The fast-scanning mode is used for searching the absorption lines of less known, or even completely unknown species, whereas the frequency-stabilized mode is used for highly-precise measurements $(10 \mathrm{kHz}$ accuracy) on selected, identified lines and for long integration times of weak signals. The technique of frequency stabilization of BWOs is well established at the Cologne 


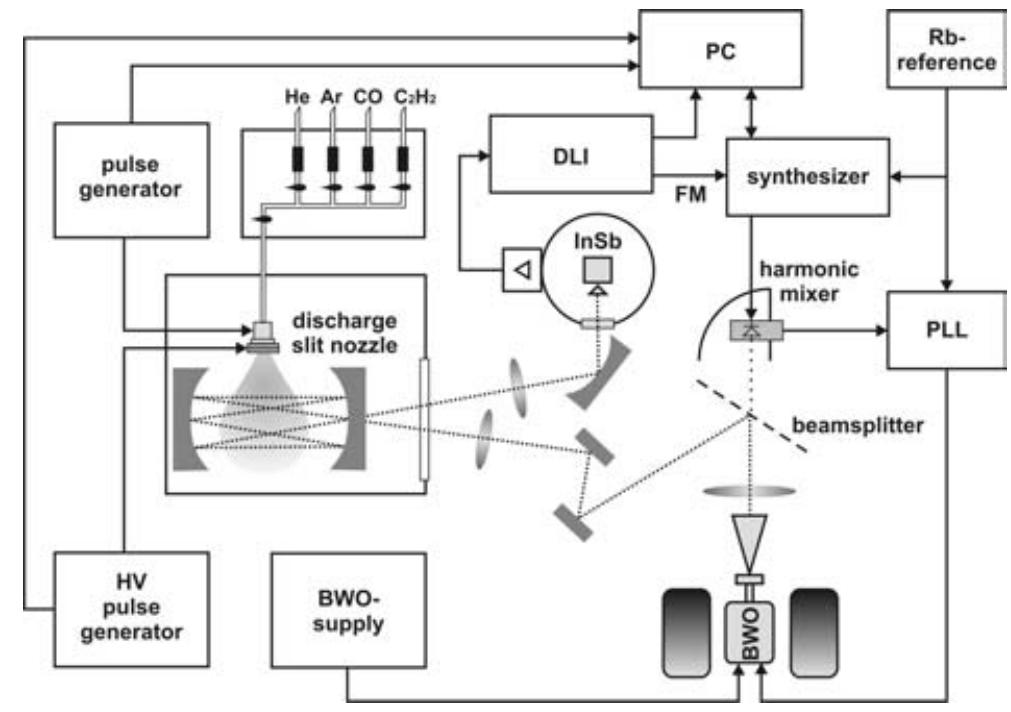

Figure 3. Experimental setup of the Cologne Supersonic Jet Spectrometer for Terahertz Applications (SuJeSTA). Backward Wave Oscillators (BWOs) are used as radiation sources. The signal is detected by a liquid helium cooled InSb-hot electron bolometer. A pulsed discharge slit nozzle produces $\mathrm{C}_{3} \mathrm{H}$ radicals in a supersonic expansion of a $\mathrm{C}_{2} \mathrm{H}_{2} / \mathrm{CO}$ gas mixture diluted in He or Ar at 1-6 bar backing pressure. The probing microwave beam intersects the molecular jet six times in a multi-pass optical array, which leads to an overall absorption pathlength of approximately $30 \mathrm{~cm}$.

laboratories (Winnewiser et al. 1994) and is used routinely in all of our measurements to achieve most reliable spectroscopic data for both laboratory and astrophysical applications.

Among a number of carbon-containing molecules detected in space, $\mathrm{C}_{3} \mathrm{H}$ is considered a key molecule of carbon-chain growth in dark clouds. There are two different isomers of $\mathrm{C}_{3} \mathrm{H}$ known, the linear $\left(l-\mathrm{C}_{3} \mathrm{H}\right)$ and the cyclic $\left(c-\mathrm{C}_{3} \mathrm{H}\right)$ forms, both of which have been identified in space by means of radioastronomy (Turner et al. 2000). The ab initio geometry for $l-\mathrm{C}_{3} \mathrm{H}$ in the ground state has been calculated to be slightly bent with a rather small barrier to linearity of $100-200 \mathrm{~cm}^{-1}$. The linear $\mathrm{C}_{3} \mathrm{H}$ radical was detected by Thaddeus et al. (1985) towards IRC+10216 and TMC-1; laboratory spectra in the ground vibrational state were observed and analyzed by Gottlieb et al. (1986). The first information on rotational transitions of vibrationally excited states was obtained by Yamamoto et al. (1990). Although they deduced the existence of an extremely low lying ${ }^{2} \Sigma$ bending state from their measurements, they were unable to directly measure transitions from the two ${ }^{2} \Pi$ electronic ground state levels into the lowest ${ }^{2} \Sigma$ bending state.

We have measured high-resolution spectra of the ${ }^{2} \Pi$ vibrational ground state of $\mathrm{C}_{3} \mathrm{H}$ and rotationally resolved transitions of the ${ }^{2} \Pi-{ }^{2} \Sigma$ bending vibration. We used SuJeSTA to perform measurements in the frequency range between 370 and $600 \mathrm{GHz}$ to extend the data set of Yamamoto et al. (1990) towards higher frequencies. A Hamiltonian for ${ }^{2} \Pi$ molecules in a Hund's case $(a)$ basis set has been used for a least squares fit analysis of all new data, including the measurements up to $350 \mathrm{GHz}$ published by Yamamoto et al. (1990). We obtained improved molecular parameters and were able to determine the exact position of the low-lying ${ }^{2} \Sigma$ band for the first time. 


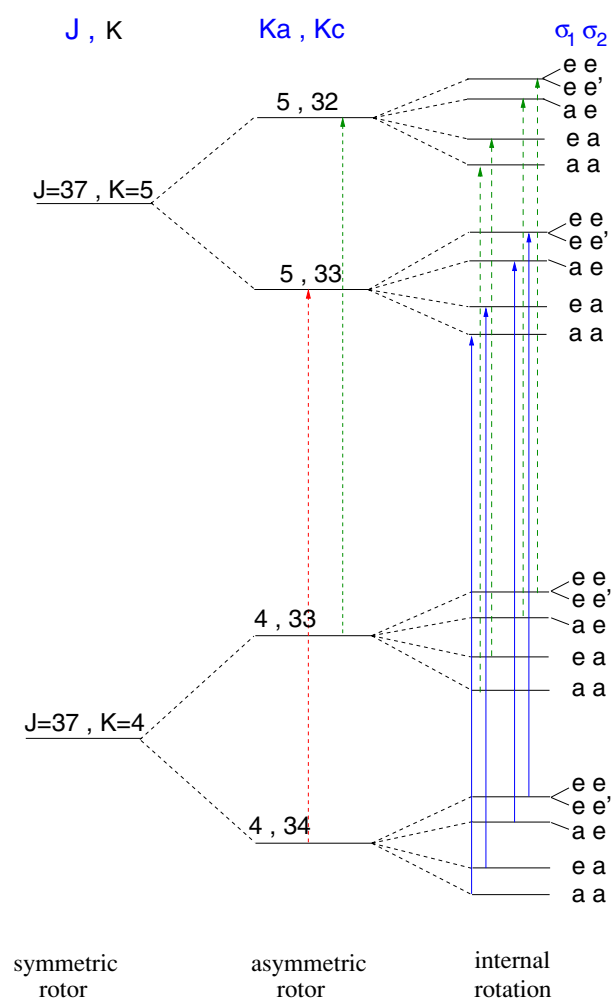

Figure 4. Energy level diagram for an asymmetric top molecule with internal rotation for the energy levels $J=37$ with $K_{a}=4,5$. Asymmetric splitting, as well as splitting due to internal rotation, is depicted.

\section{Complex Molecules - The Case of Ethyl Methyl Ether}

During the process of high-mass star formation in hot-core regions of interstellar clouds temperatures can rise to $100-300 \mathrm{~K}$ and subsequently lead to evaporation of icy mantles from interstellar dust grains (Millar et al. 1998). The mantles are thought to contain alcohols such as methanol $\left(\mathrm{CH}_{3} \mathrm{OH}\right)$ and ethanol $\left(\mathrm{C}_{2} \mathrm{H}_{5} \mathrm{OH}\right)$, which have been produced via surface reactions (Charnley et al. 1995). In the gas phase, these alcohols can react with protonated alcohols to form ethers. Dimethyl ether $\left(\mathrm{CH}_{3} \mathrm{OCH}_{3}\right)$ is the simplest representative of this class and has been found in hot cores (Ikeda et al. 2001). It is thus very likely to find larger ethers, such as ethyl methyl ether $\left(\mathrm{CH}_{3} \mathrm{CH}_{2} \mathrm{OCH}_{3}\right)$, in hot-core regions. Charnley et al. (2001) observed a single line in W51 e1/e2 and Orion KL at $160.1 \mathrm{GHz}$ and another at $79.6 \mathrm{GHz}$ in Sgr B2(N), which they assigned to trans-ethyl methyl ether. Since warm interstellar regions possess a rich interstellar chemistry, they have fairly dense spectra, and consequently these single line detections remained tentative. An unambiguous confirmation was highly desirable. We therefore started a laboratory investigation of ethyl methyl ether, using the FASSST spectrometer at Columbus, Ohio and the Cologne Terahertz spectrometer to record precise spectra from $55 \mathrm{GHz}$ to $350 \mathrm{GHz}$. Ethyl methyl ether is an asymmetric top molecule with a strong $b$-type and a weaker $a$-type spectrum. Figure 4 shows the energy levels of the $J_{K}=37_{4}$ and $J_{K}=37_{5}$ rotational states. Asymmetry splitting lifts the degeneracy of the rotational levels, and the energy level diagram is further complicated by the internal rotation of the two methyl groups, which causes each level to split into five sub-levels. 
From the analysis of more than 1000 individual lines including the lower-frequency transitions up to $40 \mathrm{GHz}$, which had been published earlier by Hayashi \& Kuwada (1975), precise molecular parameter were derived to give reliable line frequency predictions up to $400 \mathrm{GHz}$ (Fuchs et al. 2003). In 2002 and 2003, we used the IRAM 30-m telescope at Pico Veleta, Spain, and the SEST 15m telescope at La Silla, Chile, to search for ethyl methyl ether in several hot-core regions (Fuchs et al. 2005). The spectra contain lines from other organic molecules; most prominent are methanol lines, which overlap with many line positions of ethyl methyl ether. For all sources, we were able to find a set of ethyl methyl ether transition frequencies that are not blended by any other constituent. We clearly detected ethyl methyl ether towards W51 e2 and estimated a column density of $2 \times 10^{14} \mathrm{~cm}^{-2}$ at $T_{\text {rot }}=70 \mathrm{~K}$. Although these measurements confirm the tentative detection towards W51 e2 published by Charnley et al. (2001), we cannot confirm their tentative detection of ethyl methyl ether in Orion KL. In W51 e2 we also determined the relative abundance of ethyl methyl ether with respect to ethanol to be 0.13 , which indicates a high abundance of ethers.

\section{Conclusions}

Ground-based observations in the terahertz region are hampered or completely blocked by the earth atmosphere. With the advent of new telescopes such as SOFIA and the Herschel/HIFI instrument, this important spectral window will be opened up for astronomical observations. We are thus expecting a wealth of line detections that carry information on well-known but also on completely unknown interstellar molecules. These new measurements will provide a deeper insight into physical conditions of astronomical sources and their molecular inventory. Accomplishing laboratory measurements in the terahertz region will assist the identification of spectral features and will play a key role in the detection of new molecules in space. The development of highly-precise terahertz spectrometers is thus of essential importance. New multiplier techniques based on superlattice devices will expand the spectral range to several $\mathrm{THz}$. In addition, monochromatic radiation sources such as quantum cascade lasers, which are commercially available for the mid-infrared region, will reach to the far-infrared region in the near future.

The Cologne spectrometers are well suited for the investigation of highly-reactive molecules, radicals and molecular ions in the terahertz region. Here we have presented three categories of molecules, which are of astrophysical importance. Light hydrides are thought to occur early in the formation of interstellar molecules and are appropriate candidates to test chemical models of ion-molecule and grain-gas phase reactions. Especially for di-hydrogenated species in cold environments the ortho/para ratio can deviate from the one expected from spin statistics and gives clues about the molecular formation.

Carbon-chain molecules are abundant in cold interstellar clouds and have been found in the shells of late type carbon stars. It is a challenging task to find pure carbon chains in theses objects. Although they have been predicted from chemical networks, they have so far eluded astronomical detections owing to the lack of laboratory data. We will use the Cologne spectrometers to study the terahertz spectra of pure carbon chains in the laboratory and will give precise data for their detection in space. Not only pure carbon chains, but all centro-symmetric chain molecules such as $\mathrm{HC}_{n} \mathrm{H}, \mathrm{OC}_{n} \mathrm{O}$, and $\mathrm{NC}_{n} \mathrm{~N}$, $(n=3,4, \ldots)$ have no permanent electrical dipole and thus no pure rotational spectrum. However, they have their lowest bending modes in the terahertz region, as has been predicted from ab initio calculations (Botschwina 2005).

Interstellar carbon chemistry bears a variety of complex molecules, which usually have quite complicated spectra spread over a wide frequency range. Laboratory data are rather 
sparse, but highly demanded for a more complete inventory of complex molecules in space. The Cologne spectrometers are well suited to record spectra over a wide frequency range from $50 \mathrm{GHz}$ to $2.7 \mathrm{THz}$ and will be used to investigate molecules such as dimethyl ether, ethyl formate, and ethyl cyanide at high frequencies.

Laboratory spectroscopists and astrophysicists use different languages when they talk about molecules. While spectroscopists use parameters and constants to describe molecules, astrophysicists prefer frequencies and line positions. The Cologne spectroscopy group has therefore created the Cologne Data Base for Molecular Spectroscopy (CDMS), as a link between laboratory spectrocopy and astronomy. The CDMS, which contains line catalogs of more than 300 species, uses the same format as the JPL catalog. Free access is granted via internet http://www.cdms.de.

\section{Acknowledgements}

The present investigations were supported by the Deutsche Forschungsgemeinschaft under the contracts DFG-SFB 494 and LEA HiRes.

\section{References}

Adamkovics, M., Blake, G.A., \& McCall, B.J. 2003, Ap. J. 595, 235

Bernath, P.F., Hinkle, K.W., \& Keady, J.J. 1989, Science 244, 562

Botschwina, P. 2005, Mol. Phys. 103, 1441 and references therein

Brünken, S., Michael, E.A. Lewen, F., Giesen, T., Ozeki, H., Winnewisser, G., Jensen, P., \& Herbst, E. 2004, Can. J. Chem. 82, 676

Brünken, S. 2005, Ph.D. thesis, High Resolution Terahertz Spetctroscopy on Small Molecules of Astrophysical Importance (Göttingen: Cuvillier Verlag)

Brünken, S., Müller, H.S.P., Lewen, F., \& Giesen, T.F. 2005a, J. Chem. Phys. 123, 164315

Bunker, P.R. \& Langhoff, S.R. 1983, J. Mol. Spect. 102, 204

Cernicharo, J., Goicoechea, J.R., \& Caux, E. 2000, Ap. J. 534, L199

Charnley, S.B., Kress, M.E., Tielens, A.G.G.M., \& Millar, T.J. 1995, Ap. J. 448, 232

Charnley, S.B., Ehrenfreund, P., \& Kuan, Y.-J. 2001, Spectrochim. Acta A 57, 685

Clegg, P.E., Ade, P.A.R., Armand, C. et al. 1996, A\&A A 315, L38

Douglas, A.E. 1951, Ap. J. 94, 466

Fuchs, G.W., Fuchs, U., Giesen, T.F., \& Wyrowski, F. 2005, A\&A 444, 521

Fuchs, U., Winnewisser, G., Groner, P., De Lucia, F. C., \& Herbst, E. 2003, Ap. J. Suppl. 144, 277

Gendriesch, R., Lewen, F., Winnewisser, G., \& Müller, H.S.P. 2001, J. Mol. Struct. 599, 293

Gendriesch, R., Pehl, K., Giesen, T., Winnewisser, G., \& Lewen, F. 2003, Z. Naturforsch. 58a, 129

Giesen, T.F., Van Orden, A., Hwang, H.J., Fellers, R.S., Provencal, R.A., \& Saykally, R.J. 1994, Science 265, 756

Giesen, T.F., Van Orden, A.O., Cruzan, J.D., Provencal, R.A., Saykally, R.J., Gendriesch, R., Lewen, F., \& Winnewisser, G. 2001, Ap. J. 551, L181

Gottlieb, C.A., Gottlieb, E.W., Thaddeus, P., \& Vrtilek, J.M. 1986, Ap. J. 303, 446

Hayashi, M. \& Kuwada, K. 1975, J. Mol. Struc. 28, 147

Herzberg, G. \& Shoosmith, J. 1959, Nature 183, 1801

Hinkle, K.W., Keady, J.J., \& Bernath, P.F. 1988, Science 241, 1319

Hollis, J.M., Jewell, P.R., \& Lovas, F.J. 1989, Ap. J. 346, 794

Hollis, J.M., Jewell, P.R., \& Lovas, F.J. 1995, Ap. J. 438, 259

Huggins, W. 1881, Proc. R. Soc. London 33, 1

Ikeda, M., Ohishi, M., Nummelin, A., Dickens, J.E., Bergman, P., Hjalmarson, A., \& Irvine, W.M. 2001, Ap. J. 560, 792

Jensen, P. \& Bunker, P.R. 1988, J. Chem. Phys. 89, 1327

Lee, H.-H., Bettens, R.P.A., \& Herbst, E. 1996, A\&A 119, 111 
Lewen, F., Michael, E., Gendriesch, R., Stutzki, J., \& Winnewisser, G. 1997, J. Mol. Spect. 183, 207

Lewen, F., Brünken, S., Winnewisser, G., Simeckova, M., \& Urban, S. 2004, J. Mol. Spect. 226, 113

Linnartz, H., Vaizert, O., Motylewski, T., \& Maier, J.P. 2000, J. Chem. Phys. 112, 9777

Lovas, F.J., Suenram, R.D., \& Evenson, K.M. 1983, Ap. J. 267, L131

Maier, J.P., Lakin, N.M., Walker, G.A.H., \& Bohlenender, D.A. 2001, Ap. J. 553, 267

Margulès, L., Herbst, E., Ahrens, V., Lewen, F., Winnewisser, G., \& Müller, H.S.P. 2002, J. Mol. Spect. 211, 211

Martin, J.M.L. \& Taylor, P.R. 1996, J. Phys. Chem. 100, 6047

Michael, E.A., Lewen, F., Winnewisser, G., Ozeki, H., Habara, H., \& Herbst, E. 2003, Ap. J. 596,1356

Millar, T.J. \& Hatchell, J. 1998, Faraday Discuss. 109, 15

Ozeki, H. \& Saito, S. 1995, Ap. J. 451, L97

Pickett, H.M., Pearson, J.C., \& Miller, C.E. 2005, J. Mol. Spect. 233, 174

Polehampton, E., Menten, K., Brünken, S., Winnewisser, G., \& Baluteau, J.-P. 2005, A\& A 431, 203

Scheuerer, R., Haeussler, M., Renk, K.F., Schomburg, E., Koschurinov, Y.I., Pavelev, D.G., Maleev, N., Ustinov, V., \& Zhukov, A. 2003, Appl. Phys. Lett. 82(17), 2826

Schmuttenmaer, C.A., Cohen, R.C., Pugliano, N., Heath, J.R., Cooksy, A.L., Busarow, K.L., \& Saykally, R.J. 1990, Science 249, 897

Sears, T.J., Bunker, P.R., \& McKellar, A.R.W. 1981, J. Chem. Phys. 75, 4731

Sears, T.J., Bunker, P.R., \& McKellar, A.R.W., 1982, J. Chem. Phys. 77, 5363

Sears, T.J., Bunker, P.R., McKellar, A.R.W., Evenson, K.M., Jennings, D.A., \& Brown, J.M. 1982a, J. Chem. Phys. 77, 5348

Thaddeus, P., Gottlieb, C.A., Hjalmarson, A., Johansson, L.E.B., Irvine, W.M., Friberg, P., \& Linke, R.A. 1985, Ap. J. 294, L49

Turner, B.E., Herbst, E., \& Terzieva, R. 2000, Ap. J. Suppl. 126, 427

Van Orden, A., Cruzan, J.D., Provencal, R.A., Giesen, T.F., Saykally, R.J., Boreiko, R.T. Boreiko, \& Betz, A.L. 1994, Proceedings of the 1994 Kuiper Airborne Astronomy Symposium, NASA-Ames Research Center

Yamamoto, S., Saito, S., Suzuki, H., Deguchi, S., Kaifu, N., Ishikawa, S., \& Ohishi, M. 1990, Ap. J. 348,363

Winnewisser, G., Krupnov, A.F., Tretyakov, M.Y., Liedtke, M., Lewen, F., Saleck, A.H., Schieder, R., Shkaev, A.P., \& Volokhov, S.V. 1994, J. Mol. Spec. 165, 294

\section{Discussion}

GuÉLIN: Did you study the bending modes of $\mathrm{C}_{4} \mathrm{H}$ in the same way as you studied those of $\mathrm{C}_{3} \mathrm{H}$ ? Rotational transitions within vibrationally excited states of $\mathrm{C}_{4} \mathrm{H}$ are observered in IRC+10216 and the lines are surprisingly strong. It would be interesting to learn how difficult it is to excite these states.

Giesen: $\mathrm{C}_{3} \mathrm{H}$ was the first molecule we studied with the new SuJeSTA instrument. $\mathrm{C}_{4} \mathrm{H}$ indeed would be a very interesting molecule to study in the lowest bending mode, although the predicted frequency of $226 \mathrm{~cm}^{-1}$ or $7 \mathrm{THz}$ is too high for our spectrometers.

HeRBst: Can you help identify ethyl methyl ether in space by its internal rotation splittings?

GiEsEn: In W51 e2 and Orion KL the internal dynamics of the source cause a line broadening that is larger than the internal rotation splittings of ethyl methyl ether. But in more quiescent sources, or at higher spatial resolution, the splittings would be partially resolved and could be used as characteristic patterns to identify ethyl methyl ether lines. 